\title{
Consignas para el «despegue» de un grupo de discusión: un modelo de presentación
}

\author{
Jesús GutiérRez Brito \\ Universidad Europea de Madrid
}

\section{INTRODUCCIÓN}

Una mirada atenta a la puesta en práctica de técnicas cualitativas como el grupo de discusión o la entrevista en profundidad, pone de manifiesto ciertas dificultades solventadas, en el mejor de los casos, por la no siempre presente experiencia investigadora.

En el aprendizaje de metodología cualitativa se advierte con frecuencia , y no sin razón, que el investigador no cuenta con reglas, ni recetas estandarizadas de aplicación mecánica para la puesta en práctica de los instrumentos que maneja (Orti; 1989). Incluso en el supuesto de que las hubiera, estas fórmulas serían tan numerosas y diversas que el simple operar con todas ellas supondría producir lo contrario de lo que pretenden, es decir, facilitar algo así como un manual práctico o recetario de saberes hacer ${ }^{1}$ para el investigador inexperto.

No obstante, la idea de técnicas cualitativas como instrumentos de trabajo flexibles, aprendidos artesanalmente, no susceptibles de una formalización

\footnotetext{
${ }^{1}$ La tarea del investigador se asemeja en este punto al trabajo de oficio; es decir, a un tipo de tarea que va más allá de los saberes teóricos. En este sentido: «los 'saberes hacer' equivalen a lo que aquí hemos denominado las 'cualificaciones empírico-experimentales'. Incluyen la habilidad manual, la sensibilidad digital, la intuición en el manejo de la herramienta, manipulación y la transformación física de materiales específicos, piezas, etc. A diferencia de los 'saberes' sólo es posible adquirirlas en contacto directo, es decir, material y sensorial con los procesos de trabajo. Esto explica que los procesos de adquisición de estas cualificaciones se basen en gran medida en el 'ensayo y error', es decir en la observación y experimentación directa así como en la colaboración y comunicación oral con otras personas con más experiencia en los mismos y con un conocimiento detallado del trabajo. El aprendizaje conduce a la adquisición de un virtuosismo individual en procesos de trabajo muy específicos y una reproducción lenta, en muchos casos informal y personal de este tipo de cualificaciones» (Fernández;1997:228).
} 
definitivamente cerrada ni fija, se traduce con frecuencia en una aplicación práctica más o menos confusa y descuidada que paradójicamente no está a salvo del carácter mecánico antes criticado ${ }^{2}$. Desde este punto de vista, un conocimiento más expresivo que conceptual y teórico de las técnicas cualitativas puede dar lugar a un saber hacer intuitivo y contra todo sistema. Ahora bien, por muy esforzada que sea la intención del investigador, el resultado de esta puesta en práctica asilvestrada ${ }^{3}$ es la descualificación y el desprestigio de las técnicas y los esfuerzos metodologicos por fundamentarlas.

Por este motivo, y al margen de rigideces y flexibilidades asociadas al aprendizaje y aplicación de los instrumentos de corte cualitativo, es necesario presentar ciertas consignas o pautas razonadas que garanticen, si no un recetario al uso, sí el marco o estructura sobre la cual validar o invalidar las distintas acciones orientadas a poner ciertos mecanismos en funcionamiento.

En concreto, el cometido de este artículo es contribuir en lo posible a presentar y justificar algunos pasos útiles y diestros para poner en marcha un grupo de discusión o una entrevista en profundidad. Esto quiere decir que de la amplia fundamentación metodológica elaborada en torno a técnicas cualitativas ${ }^{4}$, sólo se hará mención a aquellas justificaciones que tiene que ver directa o indirectamente con los modos de actuar para la puesta en práctica del aparato técnico.

Es decir, lo que me propongo es ejemplificar dos cosas muy concretas: por un lado, la existencia de modelos pensados ad hoc para la tarea concreta de presentar un grupo de discusión; y por otro, la advertencia de que dichos modelos, sean cuales sean, no se apoyan sobre acciones arbitrarias e intuitivas, sino sobre un conjunto de consignas validadas teórica y empíricamente a partir de la experiencia y destreza del investigador.

Si nos atemos a esta comprobación, el moderador de un grupo de discusión debe manejar la situación técnica con total garantía de saber lo que hace y para qué lo hace, demostrando así un verdadero saber hacer reflexionado que, al margen de adecuaciones o adaptaciones personales, mantenga no obstante los fundamentos básicos de actuación para la acertada presentación de un grupo de discusión.

\section{LA PRODUCCIÓN DE DATOS}

El uso de la técnica de grupo de discusión, como la encuesta, supone por un lado la recogida de discursos (datos); y por otro, el análisis de dicha información.

\footnotetext{
${ }^{2}$ Realmente, la aplicación mecánica es aquella que hace sin saber cómo ni por qué, es decir, que obvia toda la parte consciente de la operación.

${ }^{3}$ Como señala A. Orti (1989) para el grupo de discusión, el hecho de que para su uso no sean necesarias intervenciones sofisticadas, llenas de complejidades técnicas, sólo manipulables por 'supertecnócratas', no excusa cierta capacidad del investigador para poner a funcionar bajo control el teatro que el grupo reproduce. Esa mínima, pero relevante, capacidad técnica es inherente a la complejidad e incertidumbre de la situación artificial que se crea.

${ }^{4}$ Hay en la bibliografía española excelentes trabajos empíricos y teóricos que fundamentan metodológicamente las técnicas cualitativas. Véase como muestra representativa Ibáñez (1979, 1986, 1989), Lucas, de A. y Ortí (1995), Ortí (1989), Alonso (1998), Canales y Peinado (1994).
} 
Ahora bien, cuando decimos recogida, en realidad debemos decir producción. Lejos de cultivarse o recolectarse ${ }^{5}$, la información obtenida a través de una técnica es re-producida artificialmente en su totalidad por las condiciones creadas para ese fin concreto. En este sentido, los discursos son antes que nada «construcciones metodológicas» (Orti; 1989:173); y en un sentido operario más sintético son el resultado del proceso de funcionamiento de un instrumento técnico que en este caso es el grupo de discusión. Como instrumento de producción, su objetivo principal es facilitar y alcanzar la información necesaria; no obstante conviene tener en cuenta que tras este empeño el grupo imprime su impronta, y respalda la fiabilidad del discurso a nivel de funcionamiento.

Sólo un correcto operar con el grupo garantiza un uso fiable de los discursos que él mismo produce. Es más, aún a riesgo de ser extremista, el análisis posterior, incluso los objetivos planteados en la investigación, pueden verse seriamente comprometidos por lo que a priori, en técnicas cuantitativas como la encuesta, es de obligada observancia y celo por parte del investigador; es decir, controlar el correcto desempeño de las acciones que dan lugar a la aplicación y funcionamiento del cuestionario, independientemente de lo adecuado o inadecuado que sea el planteamiento global de la investigación ${ }^{6}$.

Quizá, el hecho de no formar a propósito personal cualificado para conducir grupos ?, tal como se forman entrevistadores para pasar cuestionarios, se deba, entre otros motivos, a que los primeros no relegan esta tarea a un tercero que no está directamente implicado en la investigación. La coincidencia entre investigador y personal entrevistador elimina toda desconfianza o recelo profesional que pueda afectar a la calidad de los datos, y por tanto, excusa en parte tomar medidas especiales para cumplir cuidadosamente con los pasos que se exigen y contribuyen a garantizar el funcionamiento del utillaje técnico.

En las encuestas, estas precauciones se traducen en controles más o menos exhaustivos del personal entrevistador. En concreto, la aplicación de una vigilancia disciplinaria radica principalmente en ciertos aspectos que escapan al control del investigador, y que son sometidos por sistema a supervisión, es decir, a la escucha, la evaluación y la corrección de procedimientos en la forma de hacer la entrevista y de relacionarse con el entrevistado (Fowler y Mangione; 1990). Se trata de controlar en los entrevistadores actuaciones como leer las preguntas tal como están escritas, reiterar (insistir) de forma no directiva ante respuestas inadecuadas, registrar fielmente las respuestas (no a discreción del entrevistador), y mantener con el entrevistado una relación interpersonal neutral y sin prejuicios. También se incide en un ritmo constante de entrevista, facilitar un ejemplo o

${ }_{5}$ Acciones que entrañan todavía cierta autonomía y objetividad entre productor y producto.

6 De hecho existe la fantasía que reduce toda validez y fiabilidad de las encuestas a meros aspectos de dieta, es decir, a preguntar a quienes hay que preguntar, y de la forma en que hay que preguntarles. Según este imaginario, una encuesta esta bien hecha cuando las preguntas han sido verdaderamente realizadas, y con una total honestidad. Para este cometido hay una serie de precauciones que van desde los controles (vigilancia) de los entrevistadores, hasta los filtros para que contesten quienes deben contestar.

7 Entiéndase técnicos en dinámicas de grupo. 
modelo al entrevistado cuando lo hubiera, dar instrucciones fijas al entrevistado antes y durante la entrevista para mantener unas expectativas de desarrollo y de calidad en la misma, etc.

Para el caso del grupo de discusión, la forma de controlar el discurso producido es en buena parte resultado de las dinámicas y manipulaciones que el moderador produce por el sólo hecho de manejar las herramientas que tiene a su alcance. Estas herramientas teóricas se materializan en el manejo de una presentación y una conducción adecuada para desarrollar una determinada dinámica grupal. En este sentido, la producción del discurso es una parte tan relevante en la investigación como lo es el diseño o el análisis del material obtenido.

Es más, a un nivel estrictamente retórico, la técnica está constituida por un conjunto de procedimientos organizados, dotados de sentido aparente, cuyo significado afecta a la credibilidad del investigador y de la misma investigación. Un grupo de discusión se parece a otro grupo de discusión, pero no todos responden por igual a las expectativas formales y metodológicas exigibles. Es decir, desde un estricto punto de vista formal, el que puede adoptar un observador desconocedor de la técnica, el moderador comunica una imagen de lo que es la técnica en cualquier circunstancia o contexto. En este sentido, un proceder cuidadoso debe dejar claro que un grupo de discusión no es una reunión de quince personas, ni tampoco que la duración es indefinida, según el capricho de los reunidos, o que esas personas pueden ser sustituidas en el transcurso de la dinámica por otras equivalentes, etc. Es decir, el investigador tiene en su poder la facultad publicitaria de dar credibilidad al manejo de la técnica.

Este cometido se consigue ante todo evitando confundir la flexibilidad del manejo con la permisividad del operario. El investigador debe ser consciente de que la metodología cualitativa produce en sí misma un saludable, pero a la vez censurable, efecto de desorden que no permite, por ejemplo, la encuesta ${ }^{8}$. En cierta forma, una parte de la objetividad investigadora cualitativa se apoya en el simple ordenamiento de la ingente y desaforada información producida técnicamente. De ahí que un primer nivel de análisis del discurso tenga por cometido una ordenación temática que neutralice el margen de libertad introducido en el momento de la producción del discurso.

No hace falta explicar por qué una conversación animada es mucho más rica, pero también más caótica y desconcertante, que un examen fundado en la aplicación rigurosa del esquema alienante pregunta-respuesta. Esta diferenciación tiene un efecto contundente que debe ser tenido en cuenta, y no sólo a posteriori, es decir, a la hora de aplicar el análisis. Me refiero al hecho de observar ciertas objeciones y reticencias a usar metodología cualitativa por motivos relacionados directa o indirectamente con el carácter abierto de la práctica cualitativa. Es decir, tiene que ver con los usos y abusos cometidos a raíz de una aparente y confusa libertad de acción, tanto para el moderador como para los entrevistados. Esta es en definitiva la justificación que me permite insistir en un saber hacer

${ }^{8}$ La encuesta produce una información ordenada previamente a través de la matriz que es el cuestionario. 
que evite en lo posible una imagen distorsionada de las complejidades de la investigación cualitativa, y que a la vez garantice al investigador un proceder que valide las situaciones que él mismo produce y producen discurso.

\section{TAREAS PARA PRESENTACIÓN DE UN GRUPO DE DISCUSIÓN}

Para la realización de un grupo de discusión podemos diferenciar al menos tres tipos de tareas que se alargan en el tiempo que dura todo el ejercicio. En primer lugar, la tarea de presentar e iniciar el grupo; en segundo lugar, conducirlo por los temas u objetivos marcados para la investigación; y por último, el cierre o punto final.

De los tres momentos constitutivos del proceso de producción, el primero es en extremo delicado y marca directamente los otros dos. En términos figurativos, una presentación es algo así como un despegue después del cual puede lograrse planear hasta el final de los objetivos, o por el contrario no conseguir siquiera elevar la reunión a la necesaria altura de grupo. Con estas metáforas no quiero decir que la posterior dinámica grupal sea una parte secundaria para la puesta en marcha de un grupo de discusión. Digo más bien que una adecuada presentación del grupo es un primer paso sobre el cual se asienta sin riesgos un segundo y un tercero. En concreto, un correcto despegue garantiza todo un buen desarrollo de la compleja y confusa dinámica grupal posterior. Es más, la solidez del análisis posterior del discurso debe contemplar las deficiencias y/o singularidades acontecidas en el momento de la producción. Y esas alteraciones serán más complejas y difíciles de atajar, cuanto menos consciente sea el moderador de la repercusión de sus acciones manipuladoras en la puesta en funcionamiento del grupo de discusión.

Reconocer estas repercusiones es de gran importancia para la investigación, pero aún lo es más conocer cómo y por qué se llega a controlar esta parte puramente operaria a partir de ciertas acciones intencionales traducidas en la práctica por un modelo de presentación del grupo.

Estas acciones manipuladoras se fundan a su vez en presupuestos teóricosexperimentales tomados de la psicología, en especial del psicoanálisis, y de la teoría de roles. Tanto desde un punto de vista psicológico como sociológico, lo que denominamos despegue se corresponde indistintamente con la necesidad de explicitar un contrato comunicativo, es decir, dar a conocer unos saberes mínimos compartidos que «constituyen la base común de los primeros intercambios entre entrevistador-entrevistado, y que se funda en los objetivos de la investigación, del cómo, por qué y quién la realiza» (Alonso;1998:82).

Sobre estos particulares, y en concreto sobre el desconocimiento recíproco de las partes sobre la nueva situación forzada ${ }^{9}$, se funda una relación comunicati-

9 Tal como apunta Lindlof (1995), es muy probable que los participantes para una entrevista tengan definiciones propias de lo que es un encuentro de ese tipo, y lo que supone ser entrevistado. Existe en cada uno de ellos la idea de roles y propositos consolidados que seguramente no se ajustan por experiencia propia -en la escuela, el trabajo, los medios de comunicación, etc.- a una situación de ese tipo. 
va donde: por un lado, el moderador debe llegar a formar, controlar y dirigir al grupo; y por otro, el grupo debe poder controlar y dirigir el guión (la entrevista) del moderador.

Este tipo de relaciones se fijan y controlan con una presentación introductoria que contemple lo que en la entrevista psicológica es denominado como encuadre, es decir, con una intervención técnica donde un conjunto de variables se transforman en constantes (Bleger; 1984). La finalidad del encuadre no es otra que la de delimitar y estandarizar la situación comunicacional entre moderador y grupo con la intención de conocer con mayor alcance y garantía lo que en dicha situación se produzca.

Siguiendo las observaciones anteriores, la presentación de un grupo de discusión debe fijar al menos tres tipos de variables: las comprendidas en torno a la actitud técnica y rol del moderador, las referidas a los objetivos de la reunión, y las variables tiempo y espacio donde se produce la acción. Veamos con más detenimiento cada uno de estos apartados.

\subsection{Actitud técnica y rol del moderador}

Es quizá la parte más difícil de sintetizar y de poner en práctica. Las personas reunidas para formar parte de un grupo de discusión no sólo no se conocen entre sí, sino que a demás desconocen la nueva situación en la que se encuentran ${ }^{10}$. Este peculiar contexto, y la consecuente ansiedad que genera, debe ser aprovechado y resuelto técnicamente por el moderador. Es más, una situación general de ansiedad es con lo que cuenta el saber técnico para, en primer lugar, encontrar su propia demanda; y en segundo lugar, disponer de una atención incondicional por parte de los asistentes. Es decir, el moderador puede y debe en este momento mostrarse sin confusión alguna como la persona capaz de conocer e informar de las pautas concretas para organizar la nueva relación instituida. Debe con su actitud disipar ansiedades a la vez que las instrumentaliza para motivar y crear el interés suficiente de cara al trabajo que habrá de realizar el grupo. En este sentido, toda aclaración y refuerzo narcisístico para los entrevistados acrecienta y consolida esta parte emocional orientada a facilitar la actitud técnica del investigador.

Este cometido es complementado a su vez por la declaración de roles a desempeñar por el moderador y el resto de personas que componen la reunión. El papel técnico del moderador debe ahora disociarse de forma que, por un lado, sea parte del núcleo que convoca; y por otro, permanezca al margen de dicha formación. Tal como sucede en la entrevista psicológica, el entrevistador «tiene que jugar los roles que en él son promovidos por el entrevistado, pero sin asumirlos en su totalidad» (Bleger; 1984:30). El técnico animará y expresará su decisión de implicarse como uno más en el proyecto grupal, pero por otra parte hará el esfuerzo de mantenerse fuera para saber y controlar la situación.

10 Aunque este es un requisito ideal que cada vez se cumple menos, el hecho de haber asistido anteriormente a un grupo de discusión no elimina por completo el desconocimiento asociado a un nuevo encuentro. 
En la presentación, por tanto, aparecen identificaciones entre el moderador y las personas captadas para la reunión. Por ejemplo, la forma de dirigirse al grupo en primera persona del plural va transmitiendo cierta idea de complicidad y unidad necesaria con el objetivo de crear relaciones simétricas. Las tareas que propone el moderador son las que él mismo se propone y anima a proponer a los demás. Ahora bien, sobre este rol de miembro de un grupo de iguales se apoya a su vez la explicación del rol que van a desempeñar los entrevistados desde un punto de vista técnico. El moderador pasa a ocupar una relación asimétrica, y a explicar (enseñar) el desempeño de los roles para participar en un grupo de discusión. Para ello debe dejar claro en qué consiste, y también cuál es el comportamiento esperado de alguien que participa en un encuentro de este tipo.

Una forma muy operativa de lograr este objetivo es explicar el grupo de discusión en oposición a la conocida técnica de la encuesta. Por comparación, los asistentes pronto se hacen cargo de en qué consiste la propuesta y cuál es la mecánica de su funcionamiento. No está de más introducir metáforas o ejemplos que hagan más explícitas y claras las expectativas del moderador respecto al comportamiento esperado de los participantes. De no lograrse completamente estos cometidos, la reacción más inmediata nada más iniciarse el debate será la de desconcierto, no haciéndose esperar la demanda de aclaraciones como «iy qué es lo que quiere saber?, o ¿en qué le podemos ayudar?, o más directamente, ¿y cuál es la pregunta qué nos hace?, etc. La aparición de estas interpelaciones nos avisan de que es necesario volver a replantear los roles y allanar lo estropeado por una deficiente intervención técnica ${ }^{11}$. Lo ideal, y directamente vinculado a una correcta presentación, es que ningún componente del grupo pregunte, ni tenga la sensación, o necesidad, de someterse a la forma tradicional de ser preguntado, es decir, al modo examen.

Hay no obstante, algo más que indicar en torno a los roles desempeñados en un grupo de discusión. Me refiero en concreto a otros sujetos u objetos que estando presentes en la reunion no alcanzan la dominancia y el protagonismo del moderador y de los integrantes del grupo. Es muy posible que otra persona (investigador o cliente) desee asistir a la reunión en calidad de oyente, y es seguro también que el moderador cuente a demás con algún objeto (grabadora o vídeo) que le permita registrar cómodamente la reunión. Si esto sucede, en la presentación debe explicitarse tanto su presencia como su papel en la sala. El motivo tiene que ver, no tanto con la necesidad de que estos acompañantes conozcan y aprendan su papel, como de que no sigan generando a los asistentes paranoias y ansiedades innecesarias ${ }^{12}$. Por este motivo, en toda presentación deberá existir una mención

"Hay que diferenciar estas interpelaciones fruto de la incertidumbre aún no resueltas por la presentación, y las interpelaciones referidas a la inseguridad de los participantes y su relación de dependencia con el moderador. Sólo en este caso, las primeras intervenciones interrogan al moderador para asentir y tener la seguridad de que se ha comprendido y se está haciendo bien.

12 «El magnetofón y/o el vídeo son memorias auxiliares: registran los componentes temporales y/o espaciales del discurso del grupo -reservas de memoria para el preceptor-. Pero juegan también un papel mítico: en cuanto son vividas imaginariamente por los miembros del grupo como dobles del preceptor» (Ibañez; 1989). 
explícita para los aparatos de registro, así como para los acompañantes (observadores) que entren a estar en la sala. Se explicará con cierto detenimiento el papel que juegan, y se pedirá permiso a los asistentes para que estén presentes, y en el caso de los aparatos reproductores, que puedan registrar la reunión. Una declaración sincera de buenas intenciones, aun a riesgo de ser desestimada, es preferible a las alteraciones y desconfianzas que produce un agente extraño en un medio a priori llamado a generar lo antes posible la unidad de los participantes.

\subsection{Objetivos de la reunión}

En toda presentación debe aparecer enunciado por el moderador los objetivos de la reunión. Esta tarea se aborda explicando los motivos que mueven la investigación o al cliente, y el tema sobre el cual va a girar la conversación. Es oportuno empezar por la identificación del moderador y de la empresa, instituto o universidad para la cual se está trabajando. La descripción y alcance del trabajo realizado es un segundo paso a cubrir para ir planteando y justificando los motivos de la reunion. Los motivos aludidos deben dejar claro a los componentes varias cosas: en primer lugar, que la intención principal del encuentro es obtener de ellos una información. Es decir, que hay un programa y un único objetivo u orden del día que es trabajar sobre algún aspecto o tema que preocupa al investigador o cliente. En segundo lugar, que dicha preocupación sea utilizada por el moderador como acicate para motivar y estimular el interés de los asistentes. Y en tercer lugar, que se les haga conocer el valor e importancia de su participación en las preocupaciones que inspiran la investigación.

En cuanto al tema, la introducción tiene que cuidar dos aspectos concretos, el primero es que no se den muchos datos al respecto, basta con una mención sucinta del tema a tratar para que no se produzca ningún tipo de implicación o proyección del moderador; y en segundo lugar, hacer la observación de que todos los participantes son buenos conocedores del tema, y que por ese motivo han sido elegidos para la reunión. Respecto a la forma de plantear el tema en cuestión, conviene tener en cuenta las necesidades estratégicas y metodológicas de la investigación. No obstante, es posible dar a conocer el tema de dos formas (Ibáñez; 1989). Una consiste en presentarlo de manera concreta para los objetivos de la investigación, es decir, focalizar desde un principio el aspecto temático que preocupa y sobre el cual se quiere centrar la reunión; la otra forma amplia dicho aspecto a un tema más genérico que permita posteriormente ir derivando la conversación del grupo hasta los derroteros que se desea tratar en profundidad.

\subsection{TIEMPO Y ESPACIO DE LA REUNIÓN}

La actuación de los miembros del grupo está sometida a unos límites temporales y espaciales de la sala. Estos límites son fijados explícitamente en la presentación a través de la comunicación verbal y no verbal. El tiempo como el espacio debe acotarse e imponerse con una intención clara de orientar a los asistentes 
al trabajo. Se pretende ante todo que el grupo sepa y recuerde que el espacio y el tiempo que ocupa está diseñado únicamente para la investigación. Con este sentido es oportuno hacer una mención concreta a la duración aproximada de la reunión, incluso agradecer por adelantado el tiempo que los participantes ceden de otras actividades u obligaciones.

Para el caso de la variable espacial, la recomendación más significativa de cara a la presentación es que el moderador pueda elegir el sitio donde sentarse a la mesa. Es decir, que establezca el lugar más idóneo para presentar la reunión y facilitar el trabajo de conducción. Para ello debe tenerse en cuenta el tipo de mesa, y la disposición de los aparatos auxiliares que utiliza el moderador, especialmente cámara de vídeo, pizarra, proyector, etc. Con la misma antelación, el lugar del acompañante, técnico o cliente, deberá ser dispuesto siempre al lado del moderador, el cual habrá dejado algún objeto que señale o marque la ocupación de esos lugares. En el desafortunado caso de contar con una mesa cuadra o rectangular, se puede aprovechar los espacios que ocupan las esquinas para facilitar la idea de círculo y la dinámica grupal, y adicionalmente evitar en lo posible la presencia de una autoridad que encabeza formalmente la reunión. Es frecuente que las reuniones se hagan en hoteles $u$ otro tipo de instalaciones no diseñadas a propósito para estos menesteres. En ese caso, puede incluirse dentro de la presentación la preparación y acondicionamiento de la sala, es decir, la elección de una mesa circular con sillas o sillones todos iguales y sin pretensiones, una ausencia o mínima decoración del ambiente, buena iluminación, etc. Todos estos detalles son parte introductoria y conformadora del trabajo inicial para una satisfactoria puesta en marcha de un grupo de discusión.

\section{UN MODELO EJEMPLO DE PRESENTACIÓN}

El conjunto de consignas referidas en el apartado anterior sirven para la fijación del encuadre de la reunión. No obstante, puede ser de gran interés práctico ver cómo dichas consignas aparecen formuladas y justificadas en un modelo concreto de presentación. Para este ejercicio transcribo una introducción estándar para un grupo de discusión, y posteriormente hago los comentarios oportunos para clarificar y desechar cualquier idea de arbitrariedad en la preparación de los contenidos expuestos. Obviamente, el modelo de presentación elegido es uno entre mil. La posibilidad de modificar, por ejemplo, ampliar o simplificar la extensión de la presentación, es parte de la experiencia técnica y adaptaciones personales que hace el moderador. No obstante, ciertos parámetros han de verse reflejados necesariamente en el modelo que personalicemos. La siguiente presentación consigue este mínimo exigible:

Buenos días a todos. Antes que nada quiero agradecerles a cada uno de ustedes las molestias que se han tomado para venir hasta aqui. Se lo ocupados que estamos todos y el esfuerzo que esto supone, por eso les doy las gracias.

Mi nombre es $X$, y estoy trabajando para este centro de investigación de opinión pública que se llama $Y$. 
Como seguramente saben, estos centros de investigación se ocupan principalmente de recoger la opinión de la gente sobre muy diversos temas. Ahora bien, los temas son sugeridos por clientes que están interesados en conocer lo que opina la gente sobre alguna cosa que les afecta o preocupa muy directamente, por ejemplo el funcionamiento de un servicio, la venta de un producto o el agrado de una imagen.

Esos clientes son de dos clases principalmente: un primer tipo de cliente lo forman instituciones oficiales como la Administración Pública, es decir, Ayuntamientos, Comunidades Autónomas y Ministerios. Otro tipo de cliente, está vez privado, son los fabricantes, las agencias de publicidad, los bancos, los partidos políticos, etc.

Son ellos los que nos encargan las investigaciones, y es para ellos para quienes hacemos el trabajo de preguntar la opinión que tiene la gente sobre diversos temas que les preocupan.

¿Cómo preguntamos la opinión del público? Pues la forma más habitual y conocida es a través de las encuestas. Seguramente todos ustedes ya saben lo que es una encuesta aunque solo sea por los periódicos o porque posiblemente ya han tenido que contestar a una. ¿Me confundo?

Bien, pues la encuesta es una forma concreta de preguntar a la gente por lo que opina. La forma de preguntar consiste en que yo hago una pregunta y ustedes eligen una posible respuesta. Voy a ponerles un ejemplo:

- ¿Usted fuma?: dígame sí ó no (contesta un componente del grupo al azar)

$-S t$

- Eso es. Yo hago una pregunta y usted ha elegido una respuesta; esa es la forma de preguntar en una encuesta.

Ahora bien, lo que sucede es que a veces no hay preguntas que hacer porque sencillamente no sabemos qué es lo que hay que preguntar, ni sobre qué preguntar. Sencillamente no hay preguntas.

$O$ bien sucede también, que la pregunta que yo les puedo hacer no se contesta tan fácilmente con un si o con un no, sino que se contesta con un depende del momento o de la situación que tengamos en la cabeza.

Pues bien, cuando no sabemos que preguntas hacer, o cuando no hay respuestas concretas que dar porque depende de circunstancias y situaciones variables que hay que explicar, la forma que utilizamos para preguntar la opinión a la gente es esta que ven ustedes aquí.

Reunimos en torno a una mesa un número aproximado de 8 o 10 personas para que, a título de tertulia, y de manera totalmente informal, hablen libremente sobre un tema concreto.

Lo que les pido ahora es precisamente eso, que tal como si estuviéramos en una charla de sobremesa me presten su ayuda para que, durante una hora y media aproximadamente, le demos vueltas a un tema que todos ustedes conocen bien y por el cual nos vemos reunidos.

El fin principal de esta reunión es recoger todas y cada una de las valiosas opiniones que ustedes puedan darnos. Para nosotros todo lo que ustedes puedan opinar o pensar es de gran importancia. No hay por tanto unas opiniones más importantes que otras, ni un punto de vista mejor que otro. Nos interesa todo lo que a lo largo de esta reunión puedan decirnos sobre el tema que seguidamente voy a poner sobre la mesa. 
Tengo algo más que decirles, como pueden ver sobre esta mesa tengo una grabadora y de tras de mí hay una cámara de vídeo que puede pasar imágenes de esta reunión a otra sala donde se encuentra un compañero mío y el cliente que ha encargado la investigación, y que desea verla.

Yo les voy a pedir antes de empezar que me den permiso para poner en funcionamiento estos aparatos y grabar las conversaciones. No tiene mayor importancia, el motivo es que aunque yo intente coger notas de todo lo que se vaya diciendo, a veces, como hablamos muy de prisa y varios a la vez, no puedo apuntar todo lo que dicen, y tal como lo dicen. Por eso estos aparatos son para nosotros tan importantes, porque permiten después de la reunión, y y a tranquilamente, ir repasando todo otra vez sin temor a la interrupción y sin el apremio del tiempo.

Bueno, pues si están preparados podemos pasar a presentar el tema sobre el cual vamos a hablar. En este caso se trata de hablar de X. Cualquiera de ustedes puede, cuando quiera, romper el hielo. Ahora ya sólo les pido que me den permiso para poner a funcionar los aparatos. ¿Me permiten grabar la conversación y conectar la cámara de vídeo? Gracias. ¿Quién empieza? (...)

El ejemplo de presentación propuesto está diseñado para el caso de investigación comercial ${ }^{13}$. El lenguaje trata de ser accesible a todo tipo de público, algo que debe combinarse adecuadamente con una cuidadosa organización didáctica de las ideas expuestas para encauzar la dinámica posterior. Es importante no olvidar que uno de los objetivos principales de toda presentación es enseñar el funcionamiento de la técnica y los roles desempeñados por los asistentes. Por este motivo, el modelo reseñado se ordena secuencialmente en los siguientes pasos:

1. Una presentación del moderador, de la empresa o institución a la que representa y de la instancia que demanda la investigación.

2. Una explicación de la técnica o técnicas utilizadas para investigar el encargo o tema objeto de la investigación

3. Una demanda concreta de participación de los asistentes en el desarrollo de la investigación.

4. Una presentación justificada de los objetos y/o sujetos observadores de la reunión.

5. Un propuesta de tema y planteamiento para la inmediata discusión.

En cualquiera de estos pasos puede el moderador proporcionar refuerzos narcisistas a los participantes. Puede hacerlo agradeciendo su presencia, remarcando la importancia del tema que vamos a tratar, o más directamente ensalzando el valor de la información que estos poseen y la ayuda que nos prestan.

I3 Quiero agradecer este modelo de presentación a la empresa de Investigación de Mercados Metra-Seis, y en concreto a las sabias directrices y certeros consejos de José Luis Alvarez, Director del Departamento de Investigación Cualitativa. 
Todos los pasos están a su vez orientados a disminuir la ansiedad y la desconfianza producida por la situación inicial. Desde el principio se informa de dónde están los asistentes, con quién y por qué motivos. Como se advierte en el caso ejemplo, no hace falta dar más señas que las imprescindibles para hacerse una composición de lugar ${ }^{14}$. No obstante, es importante ir aclarando el rol de moderador respecto a la instancia que encarga la investigación. Con este movimiento se logra dos cosas: la primera, poner al moderador en una situación de mediación técnica; segunda, introducir la labor técnica (rol) del investigador.

Dicho esto, la forma de abordar con éxito este particular es a través del uso práctico de la pregunta. Recabar información a través de la pregunta es una forma rápida y cómoda de explicar en qué consiste el trabajo técnico del investigador y la dificultad básica con la que se encuentra, es decir, que en un determinado momento no pueda o no tenga preguntas que hacer a la gente. De esta forma, por defecto, se evita la expectativa del examen a la vez que se da paso a una forma distinta de preguntar que entronca directamente con la presentación del rol a desempeñar por los asistentes. La forma de enseñar este nuevo rol pasa por explicar previamente en qué consiste esa otra forma de preguntar (igual que se hace para la encuesta). En este caso describir un grupo de discusión como una tertulia, o una charla informal, permite garantizar tanto las relaciones simétricas entre los asistentes, como un ejemplo modélico de comportamiento ante el moderador.

En efecto, visto en qué consiste un grupo y los roles inferidos, seguidamente se presenta el objetivo de la reunión, es decir, explicar lo que se espera obtener. Obviamente, la idea es poner sobre aviso a los participantes de que sus opiniones y puntos de vista son el objetivo principal de la charla informal, pero a la vez se hace énfasis en tres puntos que acotan o limitan estas amplias expectativas: 1.- que esas aportaciones individuales tienen un ámbito grupal homogéneo (todas las opiniones tienen cabida por igual en el mismo espacio), 2.- que todas tienen una enorme importancia para los fines de la investigación (no hay unas opiniones más valiosas e imprescindibles que otras), 3.- que han de ceñirse al tema de investigación propuesto (orientarse todas las opiniones al trabajo y no a la dispersión).

Por último, se introducen los aparatos técnicos y personas que asisten a la reunión en calidad de simples observadores. El orden de presentación de los artilugios y personal adicional es en primer lugar descriptivo y en segundo lugar justificativo. Primero se mencionan los aparatos y su funcionamiento, y posteriormente se argumenta los motivos de su presencia. En este caso, la argumentación se vale de la labor técnica del moderador y la comodidad que exige la reunión. No obstante, y tras la breve justificación, es importante no olvidarse de pedir el permiso correspondiente a los participantes, si bien la forma ideal es dando por hecho que nada van a objetar a la petición.

14 No hace falta dar a conocer la identidad del cliente ni sus inquietudes concretas. Basta simplemente hablar en términos generales para ubicar y presuponer que la situación que viven los participantes se ciñe a un contexto parecido. 


\section{BIBLIOGRAFÍA}

Alonso, L. E. (1998): La mirada cualitativa en sociología, Fundamentos, Madrid.

Bleger, J.(1984): La entrevista psicológica (Su empleo en el diagnóstico y la investigación), Nueva Visión, Buenos Aires.

Canales, M. y Peinado, A. (1994): «Grupos de Discusión», en Gutiérrez, J. y Delgado, J. M.: Métodos y técnicas cualitativas de investigación en ciencias sociales, Síntesis, Madrid.

Cannell, Charles F. y Kahn, Robert, L. (1985): «Interviewing» en Lindzey G. y Aronson, E.: The handbook of social psychology, Addison-Wesley Publ. Company, London.

FOWLER, J. F. Y MANGIONE, T. (1990): Standardized survey interviewing; minimizing interviewer-related error, Sage Publ., California.

FERnÁNDEZ, A. (1997): Continuidad y ruptura en la modernización industrial en España, CES, Madrid.

IвÁÑEz, J. (1979): Más allá de la sociología. El grupo de discusión: técnica y crítica, Siglo XXI, Madrid.

- (1986): Del algoritmo al sujeto. Perspectivas de la investigación social. Siglo XXI, Madrid.

- (1989): «Cómo se realiza una investigación con grupos de discusión», en VV.-AA.: El análisis de la realidad social. Métodos y técnicas de investigación. Alianza, Madrid.

LindLOF, T. R. (1995): Qualitative communicatión research methods, Sage Publ., California.

LuCAS, DE A. y OrTI (1995): «Génesis y desarrollo de la práctica del grupo de discusión: fundamentación metodológica de la investigación social cualitativa» en Investigación y Marketing, $n .^{\circ} 47$.

ORTI, A. (1989): «La apertura y el enfoque cualitativo o estructural: la entrevista abierta semidirectiva y la discusión de grupo", en VV. AA.: El análisis de la realidad social. Métodos y técnicas de investigación. Alianza, Madrid.

\section{RESUMEN}

Este artículo trata de justificar y ejemplificar una posible presentación para la puesta en funcionamiento de un grupo de discusión. Este objetivo se alcanza por dos vías: la primera tiene como objetivo advertir al investigador de la importancia que tiene la parte de producción de discursos para la imagen y efectividad de la técnica; en concreto se pone énfasis en el papel que juegan ciertos instrumentos de manejo como es la presentación del grupo y su conducción. La segunda vía tiene como objetivo proponer ciertas pautas o maneras de hacer razonadas que permitan al moderador poner en práctica una presentación. Para ello se transcribe un modelo concreto y se comentan las exigencias mínimas que hay que cumplir para el despegue de un grupo de discusión.

\section{ABSTRACT}

The present paper analyses the introductory words for a focus group interviews. It trays to justify and give examples of it. This is done in two 
different ways: The first focuses on the importance that has for the technique to produce discourses, in particular it centres on the introduction of the group and its leading. Secondly, the present article propose some guidelines that help the driver to introduce and start the focus group. An introduction model is offered, and the minimum requirements that have to be considered to allow the group to take off. 